\section{Traumatic and Overuse Injuries of the Elbow}

With the advance of technology, MR images of the elbow provide exquisite soft tissue detail and can be obtained using a variety of clinical MR systems of varying strengths. MR is the modality of choice for evaluation of ligament, tendon, and muscle pathology of the elbow that typically results from traumatic or overuse injuries. In addition, MR is superior in evaluating bone marrow and chondral surfaces.

\section{IMAGING OF THE ELBOW}

Optimal imaging of the elbow is performed using a dedicated phased array elbow or extremity coil. The high signal-to-noise ratio (SNR) provides excellent resolution of soft tissue structures for the small surface area. Additionally, a dedicated elbow or extremity coil allows for more comfortable patient positioning, which will decrease motion artifacts.

Routine imaging of the elbow should include all three imaging planes. The recommended sequences for a high-field 1.5-T magnet are: coronal proton density (PD)-weighted and fat-suppressed $T_{2}$-weighted fast spin echo images (FSE) or short-tau inversion recovery (STIR); transverse PD-weighted and $T_{2}$-weighted images (which may be dual echo as specified in the tables below or may be done as two separate sequences); and sagittal fat-suppressed $T_{2}$-weighted images. The proton density weighted images provide optimal contrast of soft tissue for the best depiction of anatomy. The longer $T_{\mathrm{E}}$ sequences ( $T_{2}$-weighted) are critical because of their sensitivity for pathology such as marrow edema and chondral surface abnormalities, in addition to ligament, tendon, and muscle pathology such as sprains, strains, or tears. An anatomy (PD) and a pathology ( $T_{2}$-weighted) sequence in each of the three planes is ideal for full evaluation of the elbow (Edelman et al., 1996). A $T_{2}$-weighted sequence without fat suppression in one of the three planes is helpful to assist in differentiating true high-signal fluid from severe degeneration that may also appear high in signal on a fat-suppressed $T_{2}$-weighted image. When fat saturation is used, relatively light $T_{2}$-weighting (e.g., $T_{\mathrm{E}}$ of 40 to $60 \mathrm{msec}$ ) should be used for better signal-to-noise ratio and anatomic visualization. These sequences are also directly applicable for a 1.0-T machine. With lower-field scanners that may not have frequency-selective fat saturation available, an STIR sequence should be substituted, although the signal-to-noise ratio will be less. Fast or turbo spin-echo (FSE or TSE) sequences may be used to advantage over conventional spin-echo sequences, since they provide better resolution in the same amount of time with more repetitions. FSE or TSE imaging allows a shortened exam time, which may be needed with difficult or restless patients to optimize the quality of the images. The disadvantage of FSE/TSE imaging is the bright signal from fat, obscuring pathology that is negated by the companion fat-suppressed or STIR sequence in the same plane. Each of the three imaging planes may individually be the best for demonstrating specific anatomic structures; therefore, imaging in all three planes is recommended in order to view the three-dimensional anatomy and to achieve accuracy in diagnoses in an area of fine anatomic detail (Holtz et al., 1998).

\section{Additional Considerations}

In the postoperative patient, artifacts from the presence of hardware or micrometallic material are reduced by fast spin-echo sequences, due to the multiple $180^{\circ}$ pulses that distinguish them from conventional spin echo sequences. However, it is difficult to achieve homogenous fat saturation in the presence of post-surgical hardware or micrometallic artifacts. Therefore, an STIR sequence is preferred over fat-suppressed $T_{2}$-weighted images to maximize image quality. Lastly, increases in the bandwidth (i.e., read gradient strength) serve to reduce metal artifacts.

Contributed by Jana Crain and Charles Ho

Current Protocols in Magnetic Resonance Imaging (2003) A27.1.1-A27.1.10

Copyright () 2003 by John Wiley \& Sons, Inc.
UNIT A27.1

BASIC

PROTOCOL

Elbow

A27.1.1

Supplement 9 
Intra-articular saline or diluted gadolinium contrast administration for an MRI arthrogram may be helpful for visualizing intra-articular bodies in the absence of joint fluid. With saline injection, the aforementioned standard sequences are still used. In the post-diluted gadolinium contrast setting, the fat-suppressed $T_{1}$-weighted sequence (see sequence 3 ) is added. For a brief discussion of MR arthrogram, please see the introduction to, and step 1 in, the Basic Protocol in UNIT A22.2.

The elbow is best imaged in near-anatomic position with the arm by the patient's side and the forearm in supination. This positioning optimizes demonstration of the collateral ligaments and common extensor and flexor tendons in the coronal plane. Pronation of the forearm places the radio-ulnar joint at substantial rotation relative to the humeral/ulnar joint and markedly degrades imaging. Imaging in each plane should be planned from scouts in both of the other two planes and should be oriented to the elbow, not to the orthogonal planes of the body or scanner. A standard exam can be performed in approximately 30 to 45 min using a 1.5-T scanner. Mid- and low-field machines generally require 45 to $60 \mathrm{~min}$.

Table A27.1.1 lists the hardware necessary to perform the procedure, along with appropriate parameters. The available gradient strength will depend on the scanner, and the echo times given in other tables below may be varied accordingly (the smaller the gradient strength, the longer the echo time for a particular scan).

NOTE: Be sure that technologists and nurses have immediate access to any emergency equipment that may be relevant to a given study, or that may be needed for a particular patient, such as crash carts or oxygen.

\section{Set up patient and equipment}

1. Interview (screen) the patient to ensure that there are no contraindications for the MRI exam such as cardiac pacemakers, cerebral aneurysm clips, biostimulaters, metallic bodies within the orbit, and any technology sensitive to magnetic fields. Question the patient regarding medical conditions that may require emergency care or equipment, in addition to conditions that would inhibit the patient from being able to complete the exam.

Generally, standard screening forms (see APPENDIX 1) are used for all patients scanned in a magnetic resonance system.

The presence of any ferromagnetic metals may be a health hazard to the patient when he or she is inside the magnet, and will also affect the imaging. If in doubt as to the exact composition of the items, it is best to exclude patients with any metal implants; see Shellock (2001) for discussion of what implants may be safely scanned using magnetic resonance. Orthopedic hardware is not a contraindication; however, ferromagnetic and even nonferromagnetic material in this hardware will produce artifacts that may degrade the images. Adjustments to the technique may be made as discussed below.

Table A27.1.1 Equipment Parameters for MRI of the Elbow

\begin{tabular}{ll}
\hline Coil type & Phased-array extremity coil \\
Gradient coil strength & $25 \mathrm{mT} / \mathrm{m}$ (or whatever the system permits) \\
Cardiac gating & No \\
Peripheral gating & No \\
Respiratory gating & No \\
Oxygen & No \\
Motion cushions & Useful \\
Use of contrast agent & No \\
\hline
\end{tabular}


Patients may be accompanied into the magnet room by a friend or family member, who can sit in the room during the scan and comfort the patient as needed. This accompanying person must be screened as well to ensure the absence of loose metal objects on the body or clothing.

2. If the procedure is a research protocol, have the patient sign any necessary consent forms.

3. Have the patient remove all jewelry and clothing items or accessories that may contain metal and change into a gown to eliminate the presence of any metal that might be found in clothing. Remove pants due to the positioning of the arm adjacent to the hip.

4. Have the patient wash off any mascara and other makeup to avoid local tissue heating.

5. Inform the patient about what will occur during the procedure, what he or she will experience while inside the magnet, and how to behave, including the following:

a. If earplugs or headphones are used to protect the ears from the loud sounds produced by the gradients, the patient will be asked to wear these, but will be able to communicate with you at any time during the imaging.

b. Establish a method of communication with the patient that is clear to the patient using either an intercom or signaling device.

c. In order to obtain good results, the patient should not talk, and should avoid or minimize other movement, during each scan-i.e., as long as the banging sounds continue. Between scans, talking is allowed in most cases, but should be avoided when comparative positional studies are being performed; the patient will be informed when this is the case.

d. Nevertheless, the patient may call out at any time if he or she feels it necessary.

6. Tape a surface marker (a cod liver oil or vitamin E pill) over the site of pain, if localized, or over the palpable abnormality.

The cod liver oil pill shows up better on all sequences.

7. Have the patient lie supine with the forearm in supination at his or her side. Place the elbow, in full extension, into the RF coil with the antecubital fossa in the center of the coil; the patient's arm (not necessarily the body) should be straight and parallel to the scanning table; the arm will be parallel to the main field for a cylindrical magnet and perpendicular to the main field for a vertical-field magnet. Place sandbags on the arm and lateral to the arm in order to prevent potential motion of the arm.

A small-angle sponge may be placed under the hand/forearm for very slight flexion of the elbow joint, which may allow for more comfort and therefore reduce potential motion.

8. Advance the scan table until the centering light is at the center of the RF coil. Evaluate for right/left and anterior/posterior offsets and use these values for localizer setup. Advance the patient to the isocenter of the magnet.

Once this step has been performed, so as long as the patient does not move on the table, the table itself can be moved and then returned to the same position as before without jeopardizing the positioning of one scan relative to another.

9. If the patient is unable to lie still, provide an appropriate sedative.

\section{Sequence 1: Localizer}

10. Run the localizer to ensure the correct location of the elbow in three orientations using the imaging parameters in Table A27.1.2. 
Table A27.1.2 Primary Clinical Imaging Parameters for Sequence 1 (Localizer)

\begin{tabular}{ll}
\hline Patient position & Supine \\
Scan type & 2-D gradient echo \\
Imaging plane (orientation) & $\begin{array}{l}\text { Three planes (if unavailable use } \\
\text { transverse plane) }\end{array}$ \\
Central slice or volume center & Center of joint \\
Echo time $\left(T_{\mathrm{E}}\right)$ & $5 \mathrm{msec}$ \\
Repeat time $\left(T_{\mathrm{R}}\right)$ & $15 \mathrm{msec}$ \\
Flip angle $(\mathrm{FA})$ & $40^{\circ}$ \\
Fields of view $(\mathrm{FOV}, \mathrm{FOV}$ & $\mathrm{F})$ \\
Resolution $(\Delta x, \Delta y)$ & $200 \mathrm{~mm}, 200 \mathrm{~mm}$ \\
Number of data points collected $\left(N_{\mathrm{x}}, N_{\mathrm{y}}\right)$ & $0.78 \mathrm{~mm}, 1.56 \mathrm{~mm}$ \\
Slice thickness $(\Delta z)$ & $5 \mathrm{~mm}, 128$ \\
Number of slices & 9 total $(3$ per plane) \\
Slice gap & $1.5 \mathrm{~mm}$ \\
Number of acquisitions $\left(N_{\mathrm{acq}}\right)$ & 1 \\
Scan time & $23 \mathrm{sec}$ \\
\hline
\end{tabular}

A

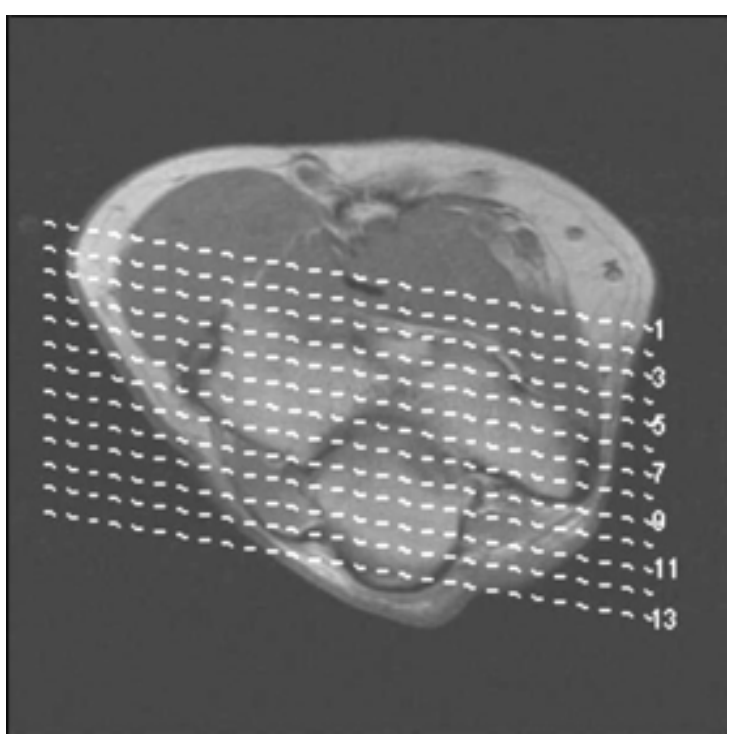

B

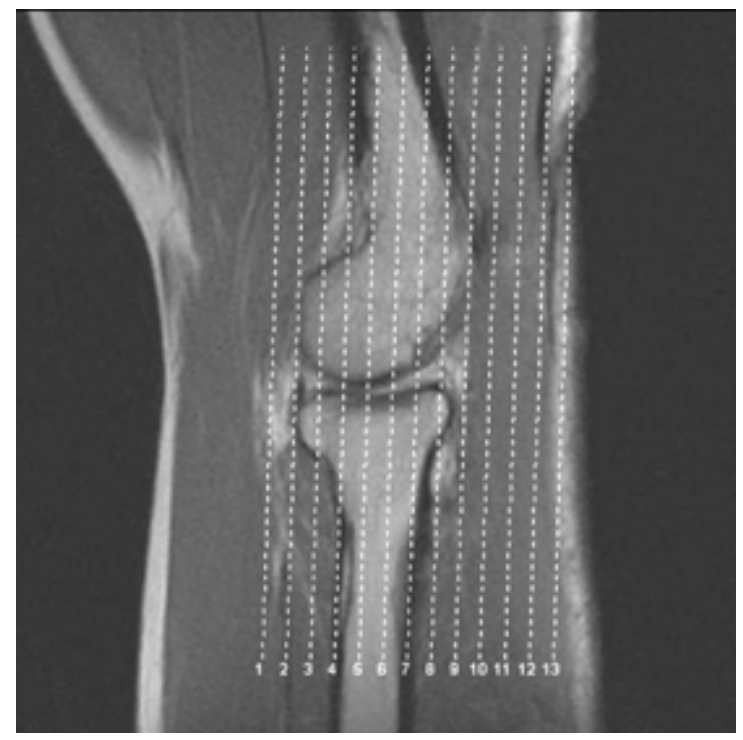

Figure A27.1.1 (A) Transverse scout for coronal images. Coronal images are obtained parallel to a line connecting the humeral condyles anteriorly. (B) Sagittal scout for coronal images. Coronal images are obtained parallel to a line along the long axis of the upper arm and forearm in the sagittal plane.

Traumatic and Overuse Injuries of the Elbow

\section{Sequence 2: Coronal proton density}

11. Use the localizer images to center at the joint line. Prescribe off transverse or sagittal scout images in order to obtain coronal images (Fig. A27.1.1).

Sample images are provided in Figures A27.1.1A and B.

12. Run sequence 2 according to the parameters in Table A27.1.3.

\section{Sequence 3: Coronal $T_{1}$-weighted (optional)}

This sequence may be substituted for sequence 2 if a shorter scan time or a $T_{1}$-weighted signal is desired (see Critical Parameters and Troubleshooting). This sequence is used if 
Table A27.1.3 Primary Clinical Imaging Parameters for Sequence 2 (Coronal PD)

\begin{tabular}{|c|c|}
\hline Patient position & Supine \\
\hline Scan type & Fast spin echo (FSE) \\
\hline Imaging plane (orientation) & Coronal oblique \\
\hline Central slice or volume center & Center of joint \\
\hline Echo time $\left(T_{\mathrm{E}}\right)$ & $33 \mathrm{msec}$ \\
\hline Echo train length (ETL) & 5 \\
\hline Repeat time $\left(T_{\mathrm{R}}\right)$ & $3000 \mathrm{msec}$ \\
\hline Flip angle (FA) & $180^{\circ a}$ \\
\hline Fields of view $\left(\mathrm{FOV}_{\mathrm{x}}, \mathrm{FOV}_{\mathrm{y}}\right)$ & $100 \mathrm{~mm}, 100 \mathrm{~mm}$ \\
\hline Resolution $(\Delta x, \Delta y)$ & $0.39 \mathrm{~mm}, 0.39 \mathrm{~mm}$ \\
\hline Number of data points collected $\left(N_{\mathrm{x}}, N_{\mathrm{y}}\right)$ & $256,256^{b}$ \\
\hline Slice thickness $(\Delta z)$ & $3.5 \mathrm{~mm}$ \\
\hline Number of slices & $16-20$ \\
\hline Slice gap & $0.4 \mathrm{~mm}$ \\
\hline Number of acquisitions $\left(N_{\text {acq }}\right)$ & 1 \\
\hline Scan time & $4 \mathrm{~min}, 53 \mathrm{sec}$ \\
\hline \multicolumn{2}{|c|}{$\begin{array}{l}{ }^{a} \text { The system displays the flip angle of the refocusing pulse. The flip angle of the first pulse of this sequence is } 90^{\circ} \text {. } \\
{ }^{b} \text { An additional } 80 \% \text { phase oversampling is applied. }\end{array}$} \\
\hline \multicolumn{2}{|c|}{$\begin{array}{l}\text { Table A27.1.4 Primary Clinical Imaging Parameters for Sequence } 3 \text { (Coronal } \\
T_{1} \text {-Weighted) }\end{array}$} \\
\hline Patient position & Supine \\
\hline Scan type & Fast spin echo \\
\hline Imaging plane (orientation) & Coronal oblique \\
\hline Central slice or volume center & Center of joint \\
\hline Echo time $\left(T_{\mathrm{E}}\right)$ & $17 \mathrm{msec}$ \\
\hline Echo train length (ETL) & 3 \\
\hline Repeat time $\left(T_{\mathrm{R}}\right)$ & $493 \mathrm{msec}$ \\
\hline Flip angle (FA) & $180^{\circ} a$ \\
\hline Fields of view $\left(\mathrm{FOV}_{\mathrm{x}}, \mathrm{FOV}_{\mathrm{y}}\right)$ & $110 \mathrm{~mm}, 110 \mathrm{~mm}$ \\
\hline Resolution $(\Delta x, \Delta y)$ & $0.43 \mathrm{~mm}, 0.54 \mathrm{~mm}$ \\
\hline Number of data points collected $\left(N_{\mathrm{x}}, N_{\mathrm{y}}\right)$ & $256,204^{b}$ \\
\hline Slice thickness $(\Delta z)$ & $3.5 \mathrm{~mm}$ \\
\hline Number of slices & 16 \\
\hline Slice gap & $0.4 \mathrm{~mm}$ \\
\hline Number of excitations (NEX) & $2^{c}$ \\
\hline Number of acquisitions $\left(N_{\mathrm{acq}}\right)$ & 2 \\
\hline Scan time & $3 \mathrm{~min}, 24 \mathrm{sec}$ \\
\hline
\end{tabular}

the gadolinium-based contrast agent is injected during the MR arthrogram, as stated in the introduction.

13. Run sequence 3 according to Table A27.1.4.

\section{Sequence 4: Coronal $T_{2}$-weighted FSE with fat suppression}

14. Run sequence 4 according to Table A27.1.5. 
Table A27.1.5 Primary Clinical Imaging Parameters for Sequence 4 (Coronal Fat-Suppressed $T_{2}$-Weighted)

$\begin{array}{ll}\text { Patient position } & \text { Supine } \\ \text { Scan type } & \text { Fast spin echo } \\ \text { Imaging plane (orientation) } & \text { Coronal oblique } \\ \text { Central slice or volume center } & \text { Center of joint } \\ \text { Echo time }\left(T_{\mathrm{E}}\right) & 44 \mathrm{msec} \\ \text { Echo train length }(\mathrm{ETL}) & 5 \\ \text { Repeat time }\left(T_{\mathrm{R}}\right) & 3000 \mathrm{msec} \\ \text { Flip angle }(\mathrm{FA}) & 180^{\circ a} \\ \text { Fields of view }\left(\mathrm{FOV}_{\mathrm{x}}, \mathrm{FOV}_{\mathrm{y}}\right) & 110 \mathrm{~mm}, 110 \mathrm{~mm} \\ \text { Resolution }(\Delta x, \Delta y) & 0.43 \mathrm{~mm}, 0.43 \mathrm{~mm} \\ \text { Number of data points collected }\left(N_{\mathrm{x}}, N_{\mathrm{y}}\right) & 256,256^{b} \\ \text { Slice thickness }(\Delta z) & 3.5 \mathrm{~mm} \\ \text { Number of slices } & 16 \\ \text { Slice gap } & 0.4 \mathrm{~mm} \\ \text { Number of acquisitions }\left(N_{\mathrm{acq}}\right) & 1 \\ \text { Fat suppression } & \text { Yes } \\ \text { Scan time } & 4 \mathrm{~min}, 53 \mathrm{sec}\end{array}$

${ }^{a}$ The system displays the flip angle of the refocusing pulse. The flip angle of the first pulse of this sequence is $90^{\circ}$.

${ }^{b}$ An additional $80 \%$ phase oversampling is applied.

Table A27.1.6 Primary Clinical Imaging Parameters for Sequence 5 (Coronal STIR)

\begin{tabular}{ll}
\hline Patient position & Supine \\
Scan type & Inversion recovery FSE \\
Imaging plane (orientation) & Coronal oblique \\
Central slice or volume center & Center of joint \\
Echo time $\left(T_{\mathrm{E}}\right)$ & $30 \mathrm{msec}$ \\
Echo train length $(\mathrm{ETL})$ & 7 \\
Repeat time $\left(T_{\mathrm{R}}\right)$ & $4100 \mathrm{msec}$ \\
Inversion time $\left(T_{\mathrm{I}}\right)$ & $140 \mathrm{msec}$ \\
Flip angle $(\mathrm{FA})$ & $180^{\circ}$ \\
Fields of view $\left(\mathrm{FOV}_{\mathrm{x}}, \mathrm{FOV}_{\mathrm{y}}\right)$ & $110 \mathrm{~mm}, 110 \mathrm{~mm}$ \\
Resolution $(\Delta x, \Delta y)$ & $0.43 \mathrm{~mm}, 0.54 \mathrm{~mm}$ \\
Number of data points collected $\left(N_{\mathrm{x}}, N_{\mathrm{y}}\right)$ & $256,205^{a}$ \\
Slice thickness $(\Delta z)$ & $3.5 \mathrm{~mm}$ \\
Number of slices & 16 \\
Slice gap & $0.4 \mathrm{~mm}$ \\
Number of acquisitions $\left(\mathrm{N}_{\mathrm{acq}}\right)$ & 1 \\
Scan time & $3 \mathrm{~min}, 45 \mathrm{sec}$ \\
\hline
\end{tabular}

${ }^{a}$ An additional $80 \%$ phase oversampling is applied.

\section{Sequence 5: Coronal STIR (optional)}

This sequence may be substituted for sequence 4 as an alternative fat-suppression sequence, e.g., for mid/low-field scanners for which frequency-selective fat-saturation fat suppression is not available, or for high-field scanners where field homogeneity is suspect and fat saturation is nonuniform, as discussed below.

Traumatic and Overuse Injuries of the Elbow

15. If this sequence is chosen to be substituted for sequence 4, run it according to the parameters in Table A27.1.6. 
A

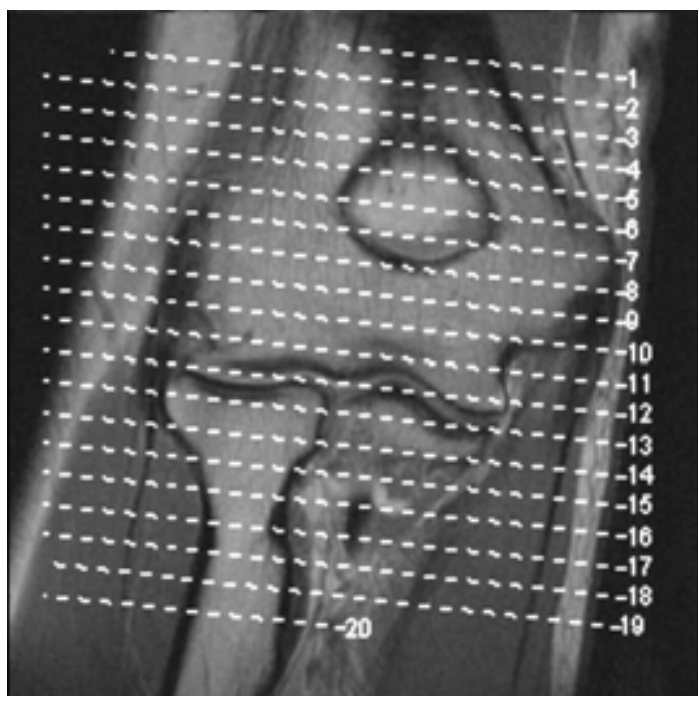

B

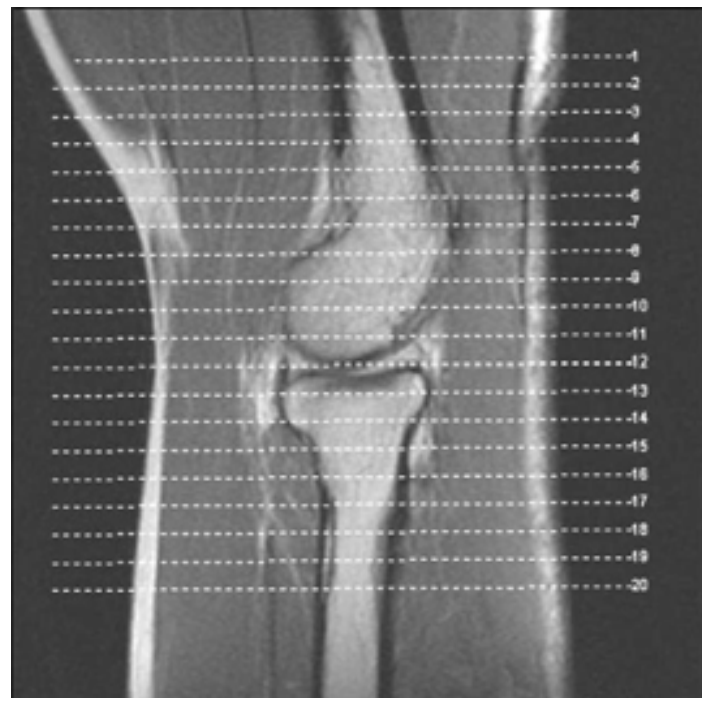

Figure A27.1.2 (A) Coronal scout for setting up transverse images. Transverse images should be obtained tangentially to the humeral condyle surface. Transverse images should extend from the distal humeral metaphysis (above the epicondyles) to the proximal radial metaphysis, notably to include the biceps attachment to the radial tuberosity. (B) Sagittal scout for planning transverse images, which should be obtained orthogonally to the long axis of the elbow in the sagittal plane.

Table A27.1.7 Primary Clinical Imaging Parameters for Sequence 6 (Transverse PD- and $T_{2}$-Weighted)

\begin{tabular}{|c|c|}
\hline Patient position & Supine \\
\hline Scan type & Dual echo PD- and $T_{2}$-weighted FSE \\
\hline Imaging plane (orientation) & Transverse \\
\hline Central slice or volume center & Center of joint \\
\hline Echo time $\left(T_{\mathrm{E}}\right)$ & $15 \mathrm{msec}$ and $92 \mathrm{msec}$ \\
\hline Echo train length (ETL) & 5 \\
\hline Repeat time $\left(T_{\mathrm{R}}\right)$ & $3000 \mathrm{msec}$ \\
\hline Flip angle (FA) & $150^{\circ} a$ \\
\hline Fields of view $\left(\mathrm{FOV}_{\mathrm{x}}, \mathrm{FOV}_{\mathrm{y}}\right)$ & $110 \mathrm{~mm}, 110 \mathrm{~mm}$ \\
\hline Resolution $(\Delta x, \Delta y)$ & $0.43 \mathrm{~mm}, 0.43 \mathrm{~mm}$ \\
\hline Number of data points collected $\left(N_{\mathrm{x}}, N_{\mathrm{y}}\right)$ & $256,256^{b}$ \\
\hline Slice thickness $(\Delta z)$ & $3.5 \mathrm{~mm}$ \\
\hline Number of slices & 20 \\
\hline Slice gap & $0.4 \mathrm{~mm}$ \\
\hline Number of acquisitions $\left(N_{\mathrm{acq}}\right)$ & 1 \\
\hline Scan time & $\sim 5 \min$ \\
\hline
\end{tabular}

\section{Sequence 6: Transverse dual echo or proton density-and $T_{2}$-weighted}

16. Prescribe off coronal or sagittal scout images in order to obtain transverse images (see Fig. A27.1.2).

17. Run Sequence 6 according to Table A27.1.7.

\section{Sequence 7: Sagittal proton density}

18. Prescribe off transverse or coronal scout images in order to obtain sagittal images (see Fig. A27.1.3). 
A

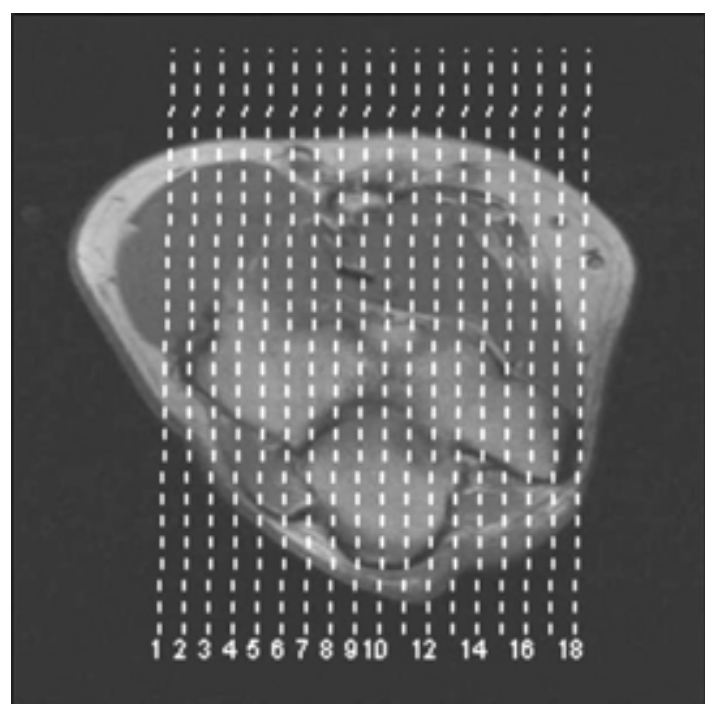

B

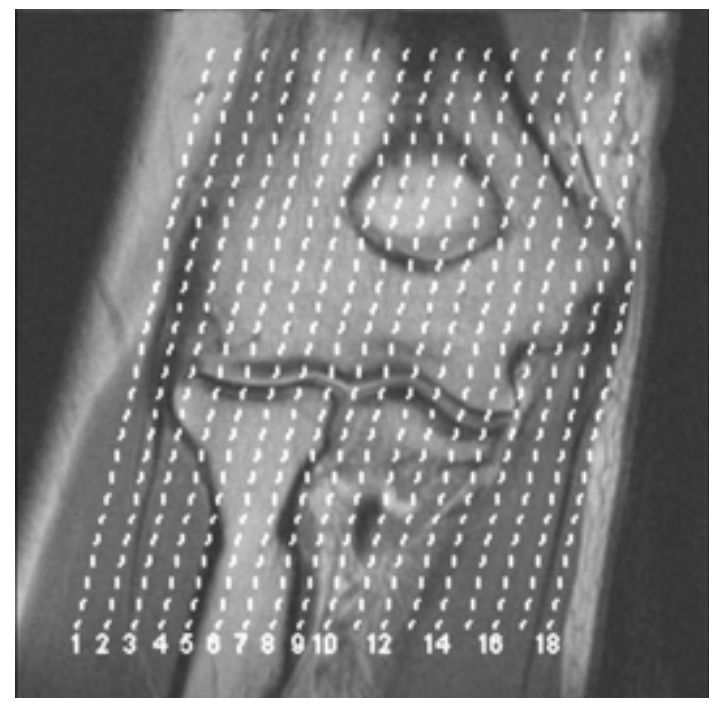

Figure A27.1.3 (A) Transverse scout for setting up sagittal images. Sagittal images are obtained perpendicularly to the coronal plane. The first and last images should include the epicondyle tips. (B) Coronal scout for planning sagittal images. Sagittal images should be parallel to the long axis of the elbow in the coronal plane of the scout image.

Traumatic and Overuse Injuries of the Elbow
Table A27.1.8 Primary Clinical Imaging Parameters for Sequence 7 (Sagittal PD)

\begin{tabular}{ll}
\hline Patient position & Supine \\
Scan type & Fast spin echo \\
Imaging plane (orientation) & Sagittal \\
Central slice or volume center & Center of joint \\
Echo time $\left(T_{\mathrm{E}}\right)$ & $33 \mathrm{msec}$ \\
Echo train length $(\mathrm{ETL})$ & 5 \\
Repeat time $\left(T_{\mathrm{R}}\right)$ & $3000 \mathrm{msec}$ \\
Flip angle $(\mathrm{FA})$ & $180^{\circ a}$ \\
Fields of view $\left(\mathrm{FOV}, \mathrm{FOV}_{\mathrm{y}}\right)$ & $110 \mathrm{~mm}, 110 \mathrm{~mm}$ \\
Resolution $(\Delta x, \Delta y)$ & $0.43 \mathrm{~mm}, 0.43 \mathrm{~mm}$ \\
Number of data points collected $\left(N_{\mathrm{x}}, N_{\mathrm{y}}\right)$ & $256,256^{b}$ \\
Slice thickness $(\Delta z)$ & $3.5 \mathrm{~mm}$ \\
Number of slices & 20 \\
Slice gap & $0.3 \mathrm{~mm}$ \\
Number of acquisitions $\left(N_{\mathrm{acq}}\right)$ & 1 \\
Scan time & $4 \mathrm{~min}, 53 \mathrm{sec}$ \\
\hline
\end{tabular}

\footnotetext{
${ }^{a}$ The system displays the flip angle of the refocusing pulse. The flip angle of the first pulse of this sequence is $90^{\circ}$.

${ }^{b}$ An additional $80 \%$ phase oversampling is applied.
}

19. Run sequence 7 according to Table A27.1.8.

\section{Sequence 8: Sagittal $T_{2}$-weighted with fat suppression}

20. Repeat step 18.

21. Run Sequence 8 according to Table A27.1.9.

The fields of view (FOV) should be adjusted to the size of the patient. The FOV values in Table A27.1.9 are suggested starting points applicable to the average-size patient. With smaller patients, the FOV should be decreased. With larger patients, the FOV can be increased if necessary. 
Table A27.1.9 Primary Clinical Imaging Parameters for Sequence 8 (Sagittal $T_{2}$-Weighted Fat-Suppressed)

\begin{tabular}{ll}
\hline Patient position & Supine \\
Scan type & Fast spin echo \\
Imaging plane (orientation) & Sagittal \\
Central slice or volume center & Center of joint \\
Echo time $\left(T_{\mathrm{E}}\right)$ & $44 \mathrm{msec}$ \\
Echo train length (ETL) & 5 \\
Repeat time $\left(T_{\mathrm{R}}\right)$ & $3000 \mathrm{msec}$ \\
Flip angle $(\mathrm{FA})$ & $180^{\circ} \mathrm{a}$ \\
Fields of view $\left(\mathrm{FOV}, \mathrm{x}, \mathrm{FOV}_{\mathrm{y}}\right)$ & $110 \mathrm{~mm}, 110 \mathrm{~mm}$ \\
Resolution $(\Delta x, \Delta y)$ & $0.43 \mathrm{~mm}, 0.43 \mathrm{~mm}$ \\
Number of data points collected $\left(N_{\mathrm{x}}, N_{\mathrm{y}}\right)$ & $256,256^{b}$ \\
Slice thickness $(\Delta z)$ & $3.5 \mathrm{~mm}$ \\
Number of slices & 20 \\
Slice gap & $0.4 \mathrm{~mm}$ \\
Number of acquisitions $\left(N_{\text {acq }}\right)$ & 1 \\
Fat suppression & Yes \\
Scan time & 4 min, 53 sec \\
\hline${ }^{a}$ The system displays the flip angle of the refocusing pulse. The flip angle of the first pulse of this sequence is $90^{\circ}$. \\
${ }_{\text {An additional } 80 \% \text { phase oversampling is applied. }}$
\end{tabular}

\section{COMMENTARY}

\section{Background Information}

The most common injuries of the elbow occur with repetitive microtrauma or acute trauma, usually during varus or valgus stress. The classic and most common clinical presentation is lateral epicondylitis, commonly known as tennis elbow. Due to overload in the varus position (backhand tennis position), there is degeneration of the common extensor tendon. Medial epicondylitis, also known as golfer's and pitcher's elbow, is secondary to overload of the flexor-pronator group occurring with repetitive valgus maneuvers. MRI findings in these conditions may include tendinosis, partial or complete tendon tears, underlying collateral ligament degeneration or tearing, and bone marrow edema. MRI is useful in identifying the presence and extent of tendon tears in addition to underlying pathology that will serve as a guide to clinical management. Fluid-filled tears are revealed nicely on longer $T_{\mathrm{E}}$ sequences $\left(T_{2^{-}}\right.$ weighted), with or without fat suppression.

Less common tendon injuries involving the biceps and triceps are well evaluated on MRI. Sagittal and transverse images are particularly useful in evaluation of these tendons. The majority of these injuries are tendon rupture. Imaging is valuable to determine the location of the tear and the distance of retraction. Sometimes the plane of imaging must be expanded to include the proximally retracted torn tendon.
Routine elbow imaging can be helpful in diagnosing the presence and etiology of neuropathies. Ulnar neuritis may be seen in the clinical setting of medial epicondylitis. Inflammation of the ulnar, radial, or median nerves commonly occurs due to entrapment or frictional trauma from adjacent anomalous musculature, bursitis, bony spurring, ganglion cysts, tumors, or fracture fragments. Inflammation of the nerve results in intrasubstance edema which is easily recognized on the fat-suppressed $T_{2}$-weighted or STIR sequences. Sequelae from neuritis may include edema and/or atrophy of the musculature due to denervation.

Contusions, fractures, stress-related edema, and osteochondral lesions may result from repetitive microtrauma or acute macrotrauma to the elbow. Osteochondral lesions may result from chronic impaction, typically lateral impaction of the capitellum. The $T_{2}$-weighted sequences are particularly useful in finding chondral surface abnormalities, as well as reactive subchondral edema. Common fractures that can be identified and further evaluated on MRI are radial head fractures in adults and supracondylar or physeal fractures in children. Anterior coronoid process fractures may be radiographically occult. Loose bodies may result from frictional trauma or acute fractures and can be identified on MR images (Sonin et al., 1996; Steinbach et al., 1997).
Elbow

A27.1.9

Supplement 10 


\section{Critical Parameters and Troubleshooting}

\section{Intra-articular bodies}

Intra-articular bodies may be difficult to visualize in the absence of a joint effusion. In this setting, intra-articular saline or dilute gadolinium administration may be helpful in localizing bodies and in evaluating the chondral surfaces that serve as donating sites. Post-contrast imaging is performed as noted above.

\section{Presence of orthopedic hardware or post-surgical/post-traumatic metallic artifact}

An STIR sequence is preferred for better homogeneity. It is difficult to obtain homogeneous fat-saturation sequences. Increasing the bandwidth also serves to reduce metal artifacts, but a notable increase may be needed, e.g., from the usual $13 \mathrm{kHz}$ to $\sim 64 \mathrm{kHz}$.

\section{Presence of a cast}

MRI can be performed in the presence of an arm cast, which may preclude the use of a flex coil. A $T_{1}$-weighted sequence (Sequence 3 ) may be substituted for the standard non-fat suppressed PD sequence to evaluate cortical margins better and to increase the conspicuity of a fracture line. Imaging of the elbow in flexion because of a cast or inability to extend can still be done. When obtaining coronal images of the upper arm, additional anterior extent of imaging will provide transverse images of the forearm. Conversely, inferior extension of transverse images through the upper arm will provide coronal images through the forearm. Center slice selection may be patient-specific but should still be chosen near the joint line.

\section{Patient positioning}

It is critical to position the arm as closely to the isocenter as possible to ensure that the elbow is within the shimming region (DSV, or diameter sphere volume) of the magnet. Have the patient shift as far as possible to the contralateral side. The contralateral arm may be flexed over the head to assist in attaining this position, if it is comfortable for the patient. If the patient is unable to shift due to his or her own size, or due to the locked position of the elbow coil, be sure that the patient's arm, but not necessarily the body, is straight and aligned parallel to the table.

\section{In the absence of a dedicated elbow coil or if the patient's arm is unable to fit in the surface coil}

A knee coil may be used as an alternative to a surface coil. The positioning of the patient changes as a result. The patient lies semiprone in the "superman position" with the arm being imaged in the extended position overhead. This is generally a more difficult position for the patient to remain in for the duration of the exam and may result in motion-degraded images. Also, in this position the forearm invariably is pronated, with resulting suboptimal anatomic demonstration in the coronal plane images.

\section{Anticipated Results}

The goals of imaging the elbow, like any other joint, include determining the source of the patient's pain, instability, or other clinical problem, as well as defining the degree of injury as well as the structures involved, which will assist the clinician in determining the appropriate pathway of management or treatment. With the development and improvement of dedicated surface coils and new techniques, MR imaging continues to be the most definitive imaging modality in evaluating soft tissue and marrow abnormalities of the elbow that result from acute and chronic injury.

\section{Acknowledgement}

Special thanks to Phillip Becklund, Dr. Rajesh Sethi, Margie Garbazino, and Susan Bybee for their time and contributions.

\section{Literature Cited}

Edelman, R.R., Hesselink, J.R., and Zlatkin, M.B. 1996. Clinical Magnetic Resonance Imaging. W.B. Saunders, Philadelphia.

Holtz, P., Erickson, S.J., and Homquist, K. 1998. MR imaging of the elbow: Technical considerations. Semin. Musculoskelet. Radiol. 2:121-132.

Shellock, F.G. 2001. MR Procedures: Health Effects and Safety. CRC Press, Florida.

Sonin, A.H., Tutton, S.M., Fitzgerald, S.W., and Peduto, A.J. 1996. MR imaging of the adult elbow. Radiographics 16:1323-1336.

Steinbach, L.S., Fritz, R.C., Tirman, P.F., and Uffman, M. 1997. Magnetic resonance imaging of the elbow. Eur. J. Radiol. 25:223-241.

Contributed by Jana Crain and Charles Ho National Orthopedic Imaging Associates San Francisco, California
Traumatic and Overuse Injuries of the Elbow

A27.1.10

Supplement 10 